\title{
Gobernabilidad y gobernanza en las Unidades de Gestión Educativa Local del Perú: una mirada desde el conocimiento de sus funciones
}

\author{
Alex Ríos Céspedes (D) @ \\ Investigador y consultor de organismos internacionales.
}

Resumen. Desde una mirada gubernamental, los órganos intermedios locales de educación requieren mejorar su eficacia y calidad. Desde una mirada de gobernanza, deberían establecer un nuevo contrato social con los actores locales. Basado en mejorar la eficacia y calidad, el presente artículo describe cuánto conocen los servidores públicos de las Unidades de Gestión Educativa Local (UGEL) sobre las funciones que realizan, y ensaya una aproximación para que pueda transitar a un esquema de gobernanza.

Palabras clave: gobernanza; gobernabilidad; competencia; conocimiento de funciones; UGEL.

\begin{abstract}
Governabilidade e governança nas Unidades de Gestão Educacional Local do Peru: Um olhar a partir do conhecimento de suas funções

Resumo. A partir de um olhar governamental, os órgãos intermediários locais de educação requerem melhorar sua eficácia e qualidade. A partir de um olhar da governança, deveriam estabelecer um novo contrato social com os atores locais. Baseado em melhorar a eficácia e a qualidade, o presente artigo descreve o quanto os servidores públicos das Unidades de Gestão Educacional Local (UGEL) conhecem sobre as funções que realizam e ensaia uma aproximação para poder transitar para um esquema de governança.
\end{abstract}

Palavras-chave: governança; governabilidade; competência; conhecimento de funções; UGEL.

Governability and governance in the Local Educational Management Unit of Peru: A look from the knowledge of their functions

Abstract. From a governmental perspective, local intermediate education bodies need to improve their efficacy and quality. From a governance approach, they should establish a new social contract with local actors. Based on improving efficacy and quality, this article describes how much the public servants of the Local Educational Management Units (UGEL) know about the functions they perform, and tests a possible route to move to a governance scheme.

Keywords: governance; governability; competence; knowledge of functions; UGEL.

\section{Introducción}

Creadas en su versión actual en el año $2002^{1}$, se puede afirmar que las 220 Unidades de Gestión Educativa Local (UGEL) existentes en la actualidad, constituyen los brazos operativos de toda política e intervención

${ }^{1}$ El Ministerio de Educación (MINEDU) crea las Unidades de Gestión Educativa como órganos desconcentrados de las Direcciones Regionales de Educación (DRE), ambos dependientes de los Consejos Transitorios de las Administración Regional (CTAR). Con el proceso de descentralización, iniciado el 2002, las DRE y UGEL pasarían a ser parte de los nuevos creados Gobiernos Regionales (GORE). Desde entonces y sin mayor regulación nacional, 16 nuevas UGEL fueron creadas. Actualmente, el número oficial de UGEL se ha definido en 220 (MINEDU, 2015a). De ellas, 213 dependen de los Gobiernos Regionales, a través de una relación directa y jerárquica con las Direcciones Regionales de Educación. Las otras 7 restantes en Lima Metropolitana constituyen órganos desconcentrados -bajo la línea jerárquica de la DRE Lima Metropolitana- del Ministerio de Educación. Existen dos tipos de UGEL, aquellas que son responsables directas de su presupuesto, usualmente 
en el sector educación, y responsables directos de asegurar que el servicio en las instituciones educativas opere de manera oportuna e ininterrumpida. Esto significa, que buscan asegurar que las instituciones educativas cuenten con docentes y materiales educativos desde el primer hasta el último día de clases, de tal manera que se cumpla el número de horas mínimas que exige el currículo para cada nivel. Si existe un evento inesperado de orden social o natural, las UGEL son las primeras en dar soporte para minimizar o recuperar el efecto en el número de horas lectivas que debe recibir un estudiante.

Aunque el rol clave de la UGEL como órgano intermedio en el servicio educativo ha sido destacado por diversos autores, se puede afirmar que hasta hoy se sabe muy poco sobre quiénes son y qué hacen, constituyéndose en una gran "caja negra” para los investigadores (Gvirtz, 2008). Este olvido, sostiene la autora, se debe en parte a la creencia que los actores de nivel intermedio son simples "correas de transmisión" de demandas y políticas entre las escuelas y los espacios de elaboración de políticas. Sin embargo, lejos de ser canales neutrales, los órganos intermedios tienen un impacto decisivo -ya sea negativo o positivo- en la redefinición de las políticas, y consecuentemente en el rendimiento del sistema educativo (Dufour, 2008).

Este parece haber sido el caso del Perú, donde las UGEL han sido órganos intermedios del sector educativo que han estado marginados de las políticas, intervenciones e investigaciones del sector. Por ejemplo, hasta el 2013 no se tenía una exacta idea del número de personal trabajando en las UGEL, menos sobre los niveles de profesionalización, competencias y conocimientos que tenían sus trabajadores ${ }^{2}$. ¿Poseen las UGEL la capacidad de satisfacer las necesidades de los ciudadanos?, ¿tienen las competencias y los conocimientos mínimos para ser eficaces?, ¿es suficiente con mejorar las competencias y conocimientos? Desde un enfoque gobernabilidad y gobernanza, el presente artículo explora el presente y las posibilidades de las UGEL. Desde una mirada de gobernabilidad, el estudio describe el conocimiento que tiene el personal de las UGEL sobre las funciones que deben realizar, encontrando variables relacionadas que permitan ofrecer recomendaciones de una política orientada a mejorar la eficacia y calidad del servicio que ofrece la UGEL. Desde una mirada de gobernanza, el artículo explora la posibilidad no sólo de mejorar la eficacia y calidad a través de reducir brechas de conocimiento, sino de establecer un nuevo acuerdo con los actores locales de la jurisdicción.

llamadas "ejecutoras" y aquellas que presupuestalmente dependen de una DRE y UGEL, usualmente llamadas "operativas".

${ }^{2}$ Recién en ese año, se realiza el primer censo de UGEL; en el 2015 se establece una tipología, se regulariza su creación, se inicia una especialización para los servidores en áreas de organización y planificación, y se financia más de mil servidores administrativos; en el 2016, se inicia la revaloración de buenas prácticas y la evaluación de los directores y especialistas pedagógicos, lo cual significó una inyección de meritocracia, remuneración y relativa estabilidad laboral. 


\section{Marco de referencia}

\subsection{Gobernabilidad y gobernanza}

Para responder los problemas de eficacia directiva de los gobiernos, la gobernabilidad y la gobernanza surgen como dos enfoques conceptuales que buscan dar solución al problema de la capacidad gubernativa del gobierno (Aguilar, 2010; 2007).

El enfoque de la gobernabilidad es estrictamente gubernamental, es decir, se refiere a la capacidad y comportamiento que tiene cada instancia de gobierno. La premisa de la gobernabilidad es la minimización de la capacidad y el rol de la sociedad: las capacidades de organización, gobierno, regulación y solución de los problemas de la sociedad son existentes o irrelevantes; todo lo contario, la sociedad es propensa al desorden, la inseguridad y el conflicto, por lo que para que la sociedad pueda ser y existir debe reorganizarse en forma de Estado y ser gobernada por una instancia dotada con los poderes, competencias, conocimientos y recursos para estar en aptitud de satisfacer sus necesidades (Aguilar, 2010; 2007).

El enfoque de gobernabilidad asocia básicamente al gobierno a la eficacia y calidad, y tiene el propósito de reforzar la capacidad operativa en la gestión gubernamental (Del Castillo-Alemán, 2011). Un gobierno capaz es suficiente para brindar servicios; por consiguiente, brindar capacidades al gobierno es la condición necesaria y suficiente para que pueda gobernar (Aguilar, 2010).

Bajo esta mirada, la receta para el enfoque de gobernabilidad consiste en brindar las capacidades idóneas y suficientes a las instancias de gobierno, a fin de que esté en condiciones de poder dirigirse en modo razonablemente eficaz y satisfacer las demandas de la sociedad (Aguilar, 2010). Aunque los esfuerzos para mejorar los servicios cubren una amplia gama de acciones que van desde cómo organizar la administración pública, hasta cómo usar tecnología de información, los resultados de las actividades públicas se deben en última instancia a los esfuerzos y el compromiso de los servidores públicos que trabajan en y para la administración pública (OECD, 2010). La evidencia empírica sugiere que uno de los principales factores institucionales que pueden mejorar el rendimiento del sector público es la implementación de prácticas apropiadas de gestión del recurso humano (Curristine, Lonti y Joumard, 2007). Por consiguiente, mejorar las competencias y los conocimientos de los servidores públicos se considera cada vez más como un aspecto esencial de la búsqueda de mejores resultados (OECD, 2010). 
El enfoque de gobernanza tiene, en la actualidad, como supuesto, que el gobierno es un agente necesario pero insuficiente, aun si tiene todas las capacidades y conocimientos requeridos; y aun si sus capacidades y conocimientos son aprovechados a cabalidad (Aguilar, 2010; 2007). Por consiguiente, se requiere de una dirección compartida entre el gobierno y las organizaciones privadas y sociales, que deben unir recursos y capacidades interdependientes para el logro de un objetivo público (Del Castillo-Alemán y Azuma, 2011). El gobierno es una instancia necesaria de dirección, pero sus ideas, acciones y recursos son insuficientes para definir y satisfacer el interés social, dado que los problemas y desafíos que la sociedad enfrenta actualmente rebasan por su magnitud, complejidad y dinámica las capacidades de respuesta que el gobierno posee (Aguilar, 2010; 2007) ${ }^{3}$.

El enfoque de gobernanza demanda un nuevo vínculo entre gobierno y sociedad; y una nueva forma de gestión pública. Una nueva relación entre gobierno y sociedad en virtud de la independencia política de los actores sociales y de su fuerza relativa de los recursos que poseen (Del Castillo-Alemán, 2012; Del Castillo-Alemán y Azuma, 2011; Aguilar, 2010 ; 2007). Aguilar $(2010,2007)$ precisa algo más: la gobernanza incluye a la gobernabilidad, en tanto se requiere la acción de un gobierno capaz y eficaz. Pero el gobierno competente es sólo uno de los actores requeridos. Este punto es importante porque gobernanza no implicaría que la sociedad ejerza o comparta los roles del Estado. La gobernanza implica más el paso de un "centro" a un "sistema" de gobierno, en el que se requieren y activan los recursos del Estado, del sector privado y de la sociedad; es el paso de un estilo jerárquico centralizado a un estilo de gobernar asociado e interdependiente entre organismos gubernamentales, organizaciones privadas y sociales (Aguilar, 2010; 2007).

En la gobernanza juega un rol importante la creación de redes como "mecanismos para facilitar la gobernanza, ya sean instancias formales o informales de información, opinión y participación activa como son las páginas electrónicas de los gobiernos, los referendos, los consejos de participación social, los observatorios ciudadanos, los movimientos sociales, entre otros" (Del Castillo-Alemán y Azuma, 2011). Para las autoras, la gobernanza es una nueva forma de gobernar lo público a través de redes políticas públicas.

3 Cabe mencionar aquí que corrientes como la Nueva Gestión Pública buscan mejorar la eficacia del gobierno; la gobernanza está orientada más a la suficiencia del gobierno. 
Si la gobernanza es considerada una aspiración para gobernar, la gobernanza local -como el espacio que administra una UGEL- es vista como el escenario ideal para el logro de objetivos específicos que sean alcanzados con eficacia y calidad, debido a las ventajas que ofrece la proximidad con el ciudadano en un espacio acotado (Del Castillo-Alemán y Azuma, 2011)

Con lo descrito, se puede afirmar que las UGEL se encuentran más orientadas bajo un enfoque de tipo gobernativo y no de gobernanza. De hecho, como afirma Del Castillo-Alemán (2011), la gobernabilidad en la gestión del sistema educativo se distingue por la búsqueda de la eficacia gubernamental. Desde los ochenta, la principal preocupación del Estado, en países como el Perú, ha estado concentrado en volver más eficiente, profesionalizada y descentralizada la gestión pública (Gonzales, Belaunde y Eguren, 2009). Parte de la búsqueda de una mayor eficacia y profesionalización estaría en la creciente preocupación por reducir las brechas de competencias y conocimientos que tienen los servidores públicos que trabajan en las UGEL.

\section{Gestión por competencias y conocimientos}

La gestión de competencias se encuentra dentro de una mirada de tipo gubernamental. Está tan extendida en el sector privado y público porque ha demostrado ser una forma eficaz de definir las habilidades y comportamientos necesarios para que las personas puedan hacer bien su trabajo (OECD, 2010). En el sector público, las competencias permiten evidenciar la capacidad de una persona para desempeñar las funciones que le son asignadas en base a los requerimientos de calidad del servicio ciudadano y resultados esperados en el sector público (SERVIR, 2016).

A pesar de su amplia difusión y extendida literatura, existe una falta de consenso sobre el significado de competencia (Emmerling y Boyatzis, 2012; Op de Beeck y Hondeghem, 2010; Nunes, Martins y Duarte, 2007). Aunque el término fue acuñado por MacClelland (1973) como un mejor factor predictivo del desempeño laboral que la inteligencia, MacClelland no definió el término competencia (García-Sáiz, 2011). Desde entonces, han proliferado definiciones y aplicaciones; por ejemplo, la mayoría de los países de la OECD utiliza su propia definición de competencia (Op de Beeck y Hondeghem, 2010; Nunes, Martins y Duarte, 2007).

Probablemente, la definición más difundida es aquella que establece las competencias como características subyacentes a las personas que están causalmente relacionadas a un criterio referenciado como efectivo o un desempeño exitoso en un puesto de trabajo (Spencer y Spencer, 1993). 
La definición es similar a la proporcionada por Boyatzis, quien describe a las competencias como características subyacentes del individuo que Ilevan o dan lugar a un rendimiento superior o eficaz (Emmerling y Boyatzis, 2012; Boyatzis, 2008; Boyatzis, 1982)4. Diversos autores han definido que tales características subyacentes conforman un conjunto de características cognitivas, afectivas, comportamentales y motivacionales que permiten un rendimiento esperado (OECD, 2010; Shirazi y Mortazavi, 2009; Gil, 2007; Alles, 2005; Emmerling y Boyatzis, 2012). Basados en Spencer y Spencer (1993), se proponen cinco características subyacentes, en base a un modelo iceberg, donde los conocimientos y las habilidades son aspectos visibles y fáciles de desarrollar, mientras que el autoconcepto, los rasgos y los motivos no son observables directamente y son más difíciles de desarrollar (Spencer y Spencer, 1993; Alles, 2005). Dado su permeabilidad y facilidad para ser enseñados, un gran número de organizaciones han destinado inversión y esfuerzo en desarrollar y evaluar los conocimientos y las habilidades del personal bajo un enfoque de competencias ${ }^{5}$.

\section{Gráfico 1. Modelo iceberg sobre competencias de Spencer y Spencer.}

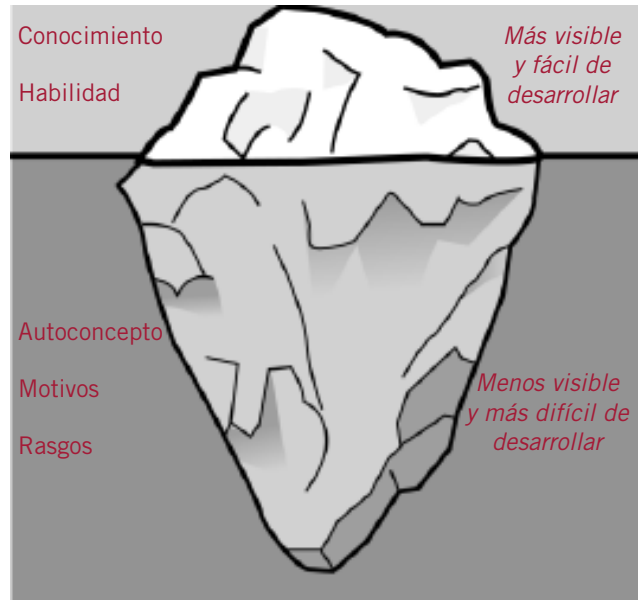

Fuente: Spencer y Spencer (1993).

${ }^{4}$ Los últimos estudios de Boyatzis combinan la definición de competencia con la definición de inteligencia emocional de Goleman para concluir que existen competencias de inteligencia emocional, social y cognitiva que se definen como la habilidad para reconocer, analizar y usar información emocional sobre uno mismo, emocional sobre otros e información general que llevan o dan lugar a un rendimiento superior. Las competencias son, sostiene el autor, una aproximación conductual de la inteligencia emocional, social y cognitiva.

${ }^{5}$ El enfoque por competencias no ha estado exento de críticas. García-Sáiz (2011) señala que existen problemas de base teórica-empírica, de definición, de evaluación, y de contexto donde se implementan. Por ejemplo, señala el autor, las competencias nacieron como una propuesta alternativa a rasgos o aptitudes, pero se definen como un conjunto de características que incluye rasgos y aptitudes; se sostiene que las competencias se pueden desarrollar, pero a la vez que ciertas características no son posibles de modificar. 
En el Perú, SERVIR (2016) define las competencias como características personales que se traducen en comportamientos visibles para el desempeño laboral exitoso en una institución pública; e involucran, de manera integrada, conocimientos, habilidades y actitudes que debe poseer y demostrar el servidor público. Dentro del marco de una gestión basada en competencias, el Estado impulsa realizar diagnósticos que permitan conocer las fortalezas y debilidades del personal público en términos de conocimientos, habilidades y actitudes (SERVIR, 2010). Los diagnósticos de conocimientos -el cual es descrito como una parte de la medición de competencias- tiene por objeto identificar la brecha de conocimientos entre las competencias descritas para el puesto y las que tienen durante el diagnóstico (SERVIR, 2010).

Es partir de los diagnósticos de conocimientos que la capacitación toma un rol importante para los servidores públicos. La capacitación debería tener como finalidad la mejora del desempeño de los servidores públicos, a través del cierre de brechas de conocimientos o competencias, para alcanzar el logro de los objetivos institucionales y de esta manera brindar servicios de calidad a los ciudadanos (SERVIR, 2010).

Sin embargo, en el país, las capacitaciones se tratan como un conjunto de capacitaciones dictadas de manera improvisada, sin ningún tipo de seguimiento o evaluación del desempeño; decididos de manera unilateral por cada sector y organizados por las necesidades de corto plazo (PCM, 2013). Además, tanto el nivel de conocimientos de las funciones como los recursos destinados para capacitaciones están distribuidos inequitativamente entre el nivel nacional y regional (SERVIR, 2015).

\section{Competencias y conocimientos en las UGEL}

Según la Ley General de Educación, la primera finalidad de la UGEL es fortalecer las capacidades de gestión pedagógica y administrativa de las instituciones educativas para lograr su autonomía (MINEDU, 2012).

A nivel de gestión administrativa, la UGEL conduce los procesos de reasignación, evaluación, promoción y cese del personal nombrado; realiza la selección y contratación del personal temporal; verifica las planillas; organiza y actualiza el escalafón de los servidores activos y cesantes tanto docente y administrativo; realiza los pagos del personal activo y pensionistas; resuelve problemas de denuncias a docentes y directivos; distribuye textos, cuadernos y material fungible desde las UGEL hasta las instituciones educativas; además paga servicios básicos de luz y agua (MINEDU, 2015b). 
A nivel de gestión institucional, la UGEL brinda asistencia técnica a las instituciones educativas para elaboración de instrumentos de gestión (proyecto educativo institucional, plan anual de trabajo, reglamento interno); supervisan y autorizan uso del mantenimiento de infraestructura; planifican, presupuestan y administran el presupuesto para cada institución educativa; brindan asistencia técnica a los directores de las instituciones educativas para mejorar la gestión institucional (MINEDU, 2015b).

A nivel de gestión pedagógica, las UGEL brindan acompañamiento o soporte técnico a los docentes por niveles, y según las intervenciones que se impulsen desde el nivel nacional o regional; son responsables de promover, asesorar y monitorear la implementación del currículo; capacitar a los docentes; impulsar y monitorear el uso de materiales educativos (MINEDU, 2015b).

Contrario a lo descrito, la gestión de la UGEL se ha caracterizado principalmente por la acumulación de labores administrativas que retrasan y dificultan la ejecución de las funciones más importantes, como ha ocurrido en las UGEL de Lima y Chincha (Valdivia y Díaz, 2008); Moquegua (Sugimaru y León, 2015); Islay en Arequipa (Vegas, 2016); y Junín (The World Bank, 2008). De acuerdo a la Defensoría del Pueblo (2013), el 72\% de los entrevistados de una muestra nacional de escuelas manifestaron, que deben trasladarse hacia su respectiva UGEL al menos 1 vez al mes; en la mayoría de casos para la realización de trámites administrativos, recogida de materiales y registro de información en el SIAGIE (Sistema de Información de Apoyo a la Gestión de la Institución Educativa).

En la práctica, lo que ha terminado existiendo es una relación inversa entre UGEL y escuela: Son los directores y docentes quienes más asisten a las UGEL para resolver diversos trámites y son menos los especialistas de las UGEL que visitan las instituciones educativas para asistirlos en temas de gestión y pedagogía. La Defensoría del Pueblo verificó que el 35\% de IIEE de una muestra nacional de 330 no fueron supervisadas por temas de matrícula (Defensoría del Pueblo, 2017); similar resultado al 2012, donde el $36 \%$ de las IIEE manifestaron que nunca fueron visitadas por la UGEL (Defensoría del Pueblo, 2013). La situación es más crítica cuando las instituciones educativas se encuentran en el ámbito rural. Los maestros en las zonas rurales experimentan una sensación permanente de abandono y olvido, y señalan no recibir suficiente apoyo de las UGEL (The World Bank, 2008). En la UGEL Julcán, La Libertad, el número reducido de especialistas es una de las causas de las pocas visitas -a veces una por año- que se realizan a las instituciones educativas (SINEACE, 2016). 
No sólo la presencia del personal de las UGEL es escasa, sino también de baja calidad o valoración por parte de los docentes. Los pocos especialistas de la UGEL que llegan a las escuelas no tienen las competencias ni los conocimientos adecuados para atender a directores y docentes. Es sintomático que en entrevistas realizadas a docentes de instituciones educativas se haya revelado una percepción negativa del desempeño de los especialistas enviados por la UGEL (Valdivia y Díaz, 2008).

Lejos e imposibilitados de reducir la carga administrativa, simplificar trámites, reorganizar la estructura o priorizar responsabilidades, las UGEL han optado por mejorar su rendimiento a través de incrementar los recursos humanos y capacitar al personal que tienen ${ }^{6}$.

Por lo tanto, no existe tanto un problema de indiferencia o ausencia de capacitación sino de eficacia de la misma. Tanto en Lima como en Arequipa, Ica o Junín, los investigadores mencionan que las UGEL hacen esfuerzos por promover la calificación del personal a través de su participación en capacitaciones, donde parte del costo de las capacitaciones son cubiertas por el propio personal (Vegas, 2016; Díaz, Valdivia y Lajo, 2008; The World Bank, 2008). El propio ministerio reporta una cobertura de 2,492 trabajadores de las direcciones regionales de educación (DRE) y UGEL capacitados durante el periodo 2012-2014, y aproximadamente 3,817 personas capacitadas durante el periodo 2015-2016 (MINEDU, 2015d; MINEDU, 2017a).

Sin embargo, la inversión en capacitaciones no correspondería con el rendimiento mostrado por el personal de las UGEL. Aunque no existen mecanismos rigurosos de seguimiento sobre el impacto de la formación de los trabajadores de las UGEL, el primer y reciente diagnóstico de conocimientos implementado entre MINEDU y SERVIR refleja deficiencias serias en el conocimiento de las funciones que deben cumplir los especialistas de las UGEL. Por ejemplo, el diagnóstico mostró que, con excepción de los especialistas de inicial, el porcentaje de conocimiento sobre las funciones que realizan no alcanza el puntaje mínimo requerido en un rango de $56 \%$ a 98\% (MINEDU, 2017b).

Bajo este marco de referencia, la siguiente sección profundiza sobre los conocimientos de las funciones que tienen los servidores públicos de las UGEL identificando factores relacionados que permitan entender con mayor profundidad el tema bajo una perspectiva de gobernabilidad.

${ }^{6}$ Las UGEL si tienen un déficit de personal pero cuyo dimensionamiento no ha sido aún sincerado o atendido por la política nacional 


\section{Metodología}

Para responder los objetivos del estudio, se obtuvo una submuestra correspondiente al Diagnóstico de Conocimientos del Personal de las DRE/GRE y UGEL Ilevado a cabo como parte de un convenio entre MINEDU y SERVIR?

La unidad de análisis es el servidor público que labora en las UGEL del país en uno de los nueve puestos de los tres principales servicios que ofrece la UGEL: acompañamiento de la institución educativa, contratación de personal docente y distribución de materiales. Los nueve puestos evaluados fueron de tipo pedagógico (inicial, primaria, secundaria ciencias y secundaria comunicación); gestión (planificación y presupuesto); y administrativo (racionalización, personal y abastecimiento).

El instrumento utilizado fue una prueba con alternativas de respuesta múltiple que fluctúa entre 66 y 114 ítems de acuerdo al puesto evaluado. Las preguntas contienen preguntas generales, específicas al puesto y situaciones de caso. El instrumento fue aplicado de manera virtual y respondida de manera voluntaria.

Cuadro 1. Tamaño de la muestra según puesto evaluado

\begin{tabular}{lcc}
\hline \multicolumn{1}{c}{ Puesto del especialista de UGEL } & $\mathrm{n}$ & Nro de preguntas \\
\hline Inicial & 203 & 66 \\
\hline Primaria & 397 & 74 \\
\hline Secundaria Ciencias & 102 & 72 \\
\hline Secundaria Comunicación & 64 & 72 \\
\hline Planificación & 142 & 114 \\
\hline Presupuesto & 152 & 90 \\
\hline Personal & 152 & 82 \\
\hline Racionalización & 105 & 67 \\
\hline Abastecimiento & 204 & 101 \\
\hline TOTAL & 1,521 & \\
\hline
\end{tabular}

Fuente: Elaboración propia.

La muestra se compone por 1,521 especialistas pertenecientes a 208 de las 220 UGEL del país ${ }^{8}$, lo que representa el $13.7 \%$ de la población total de servidores públicos de las UGEL. Al ser voluntario, se asume un sesgo en la conformación de la muestra.

${ }^{7}$ En el 2016, SERVIR se aprueba el diagnóstico de conocimientos al personal del Sistema Educativo Peruano. Nros. 010 y 011-2016-SERVIR/GDCRSC, la Gerencia de Desarrollo de Capacidades y Rendimiento del Servicio Civil

8 Las UGEL Antabamba (Apurímac), La Convención (Cusco), Pichari-Kimbiri (Cusco) y Manu (Madre de Dios) no participaron en el estudio. La UGEL Ica (Ica) fue asimilada por la DRE Ica. Especialistas de las UGEL Virú (La Libertad), Tahuamanu (Madre de Dios), Maynas (Loreto), Putumayo (Loreto), C.F. Fitzcarrald (Ancash), Lampa (Puno) y Chincha (Ica) no respondieron la sección correspondiente al diagnóstico de conocimientos. 
Para los análisis estadísticos, pruebas de distribución normal Kolmogorov-Smirnoff han sido aplicados para las variables de tipo continuo. Cuando no se encuentra normalidad, pruebas de contraste no paramétrico han sido usados para encontrar diferencias estadísticamente significativas entre dos o más grupos independientes (Siegel y Castellan, 1998) ${ }^{9}$. Para el caso de correlaciones entre las variables se usó el coeficiente de correlación Spearman, que tolera variables de tipo ordinal.

El análisis de regresión logística fue aplicado para observar un conjunto de características del personal (variables independientes) vinculadas al conocimiento (variable dependiente). La regresión logística es un tipo de regresión apropiada cuando la variable dependiente es categórica binaria, por ejemplo, la presencia y ausencia de un atributo ${ }^{10}$ (Hair et al, 1999; Johnson, 1998; Ferrán, 1996). Para ello, las categorías del puntaje de la variable conocimiento fueron reclasificadas en dos grupos, aquellos que alcanzaron por lo menos el puntaje mínimo de conocimiento sobre sus funciones y aquellos que no lo hicieron.

\section{Resultados}

\subsection{Sobre el perfil del personal de las UGEL}

En las UGEL del país predomina la presencia de varones (57\%) mientras que la edad promedio de los servidores públicos es de 46 años. Más del $80 \%$ del personal tiene una formación universitaria. El promedio de experiencia laboral es de 19 años y en el sector público es de 17 años. Sin embargo, la experiencia cae notoriamente a 4 años cuando se trata de experiencia promedio en el puesto que ocupa actualmente. Es decir, si bien los servidores públicos de las UGEL tienen amplia experiencia trabajando para el Estado, no lo es cuando se trata del puesto en que están siendo evaluados. El $88 \%$ del personal afirma que ha recibido algún tipo de capacitación (cuadro 2).

${ }^{9}$ Específicamente, se han usado los contrastes U de Mann-Whitney y Kruskall-Wallis

${ }^{10}$ La regresión logística busca obtener una combinación lineal de las variables independientes que permite estimar las probabilidades de que un servidor público pertenezca al grupo que tiene o no tiene conocimiento sobre sus funciones. La probabilidad ( $p$ ) de que un servidor público pertenezca al grupo de servidores que conoce sus funciones se da por la función. Si la probabilidad ( $p$ ) es superior o igual a 0.5 , el servidor público será clasificado en el grupo que conoce sus funciones. 
Un $26 \%$ del personal pertenece a DL 276 , de los cuales el $30 \%$ son nombrados. Un $21 \%$ está contratado bajo modalidad CAS ${ }^{11}$. Mitad del personal pertenece a la Ley de Reforma Magisterial, de los cuales el $47 \%$ se encuentra entre la cuarta y sexta escala de la carrera magisterial ${ }^{12}$.

El porcentaje de personal destacado es aproximadamente del $40 \%$, porcentaje superior al reportado por el censo de UGEL que reveló que el personal destacado alcanzaba el 33\%.

Cuadro 2. Perfil y características del personal de las UGEL

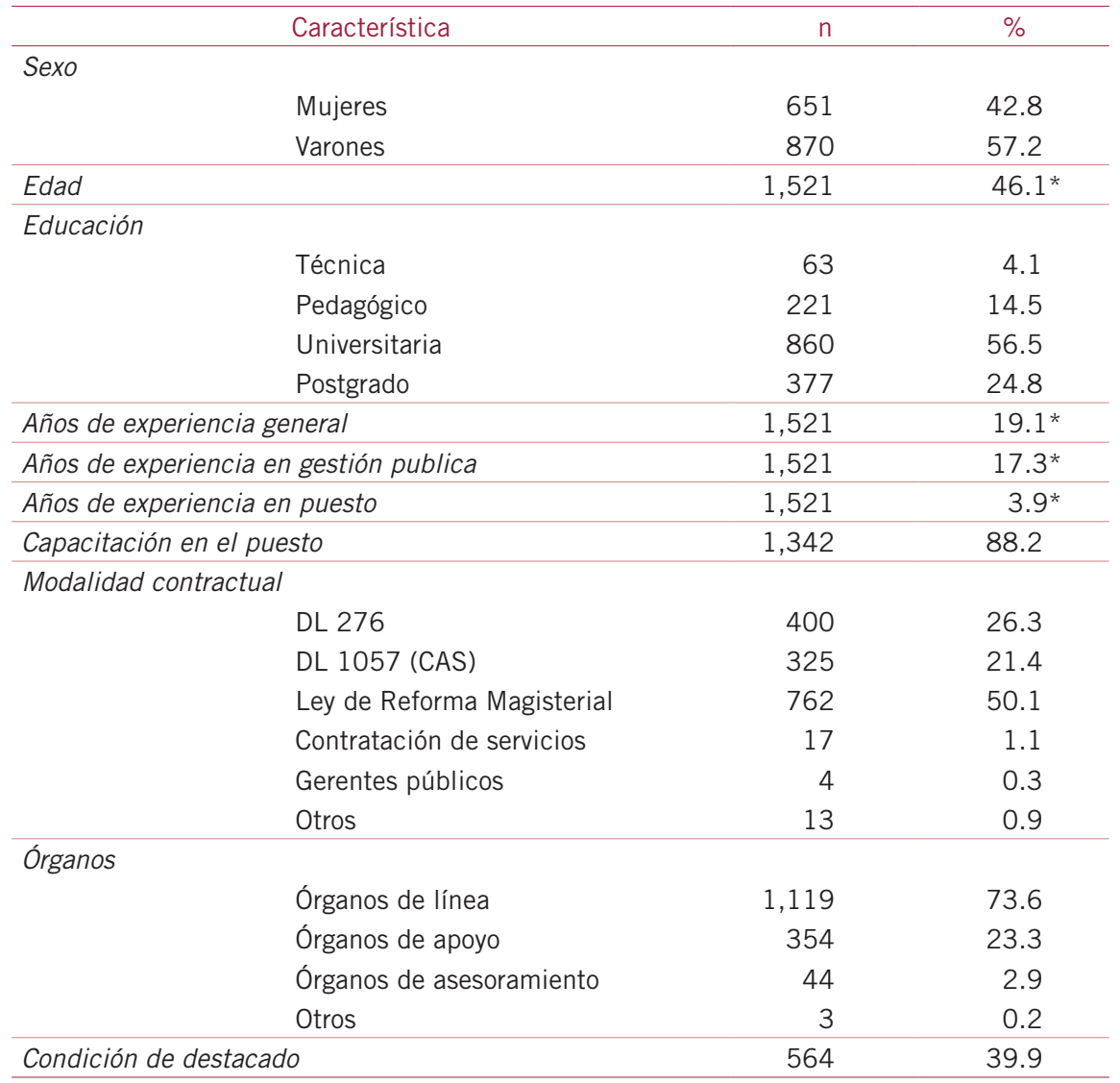

Nota: * Valores promedio.

Fuente: Elaboración propia.

${ }^{11}$ Contrato Administrativo de Servicios (CAS) es una modalidad contractual de la Administración Pública, privativa del Estado, que vincula a una entidad pública con una persona natural que presta servicios de manera no autónoma, dependiente y subordinada

${ }^{12}$ Considerar que información fue recolectada meses antes del concurso para cargos de especialistas 


\subsection{Sobre conocimientos de las funciones del personal en las UGEL}

El cuadro 3 muestra los resultados de la prueba de conocimientos respondida voluntariamente por los servidores públicos de las UGEL. El puntaje es agrupado por SERVIR en cuatro grupos según sus necesidades de capacitación. Así, quienes no alcanzan el puntaje (0-50) requieren capacitación de manera prioritaria; quienes alcanzan puntaje mínimo (51-70), necesitan capacitación en las competencias evaluadas; quienes alcanzan el puntaje esperado (71-85), requieren sólo reforzamiento; mientras los que alcanzan un puntaje destacado (85-100) cuentan con los conocimientos óptimos.

Cuadro 3. Porcentaje Promedio de Conocimientos de Funciones según Escala de Evaluación

\begin{tabular}{|c|c|c|}
\hline Resultado & $\mathrm{n}$ & $\%$ \\
\hline No alcanzó puntaje & 1067 & 71.0 \\
\hline Alcanzó puntaje mínimo & 408 & 27.1 \\
\hline Alcanzó puntaje esperado & 27 & 1.8 \\
\hline Puntaje destacado & 1 & 0.1 \\
\hline Conocimiento promedio & & \\
\hline
\end{tabular}

Fuente: Elaboración propia.

Como se observa, el conocimiento promedio fue de 41.7 en una escala de 1 al 100. De todos los evaluados, apenas el 1.8\% alcanzó el puntaje esperado. El $71 \%$ del personal cumple sus funciones sin alcanzar el puntaje mínimo de conocimiento para el puesto que viene desempeñando. Se podría decir, que casi la totalidad del personal (98\%) requiere ser capacitado en las tareas que desempeñan. Estos resultados Ilaman la atención si se considera -como puede observarse en el cuadro 2- que el personal tiene en promedio 4 años en el puesto y que el $88 \%$ asegura haberse capacitado.

Cuadro 4. Porcentaje Promedio de Conocimientos de Funciones según Tipo de Especialista

\begin{tabular}{|c|c|c|}
\hline Área evaluada & $\mathrm{n}$ & Puntaje promedio \\
\hline Inicial & 203 & 51.6 \\
\hline Primaria & 397 & 44.9 \\
\hline Secundaria Ciencias & 102 & 40.8 \\
\hline Secundaria Comunicación & 64 & 47.9 \\
\hline Planificación & 142 & 35.3 \\
\hline Presupuesto & 152 & 29.4 \\
\hline Personal & 149 & 36.1 \\
\hline Racionalización & 105 & 42.1 \\
\hline Abastecimiento & 204 & 41.4 \\
\hline
\end{tabular}

Fuente: Elaboración propia. 
De acuerdo al área donde fueron evaluados, el rendimiento es menor en las áreas administrativas y de gestión, siendo el más crítico en el área de presupuesto (29.4). El conocimiento es mayor en las áreas pedagógicas o de línea, donde destaca el nivel inicial, la única área donde el conocimiento promedio llega a 51.6 (cuadro 4).

Para definir factores asociados al conocmiento de las funciones, un modelo de regresión logística fue estimada. A través de varios pasos, introduciendo y excluyendo variables, el modelo final ofrece tres variables significativas que influyen en el conocimiento de las funciones que tienen los servidores públicos ${ }^{13}$ : ser mujer, pertenecer a la ley de reforma magisterial y haber recibido capacitación más de 90 horas. Sin embargo, nótese que el modelo explica sólo el $12.4 \%$ de la varianza de los resultados.

Cuadro 7. Coeficientes del Modelo de Regresión Logística para Conocimiento de Funciones del Personal de las UGEL

\begin{tabular}{lccc}
\hline \multicolumn{1}{c}{ Característica } & \multicolumn{1}{c}{ ( } & EE & p-value \\
\hline Mujer &, $573^{* * *}$ &, 120 &, 000 \\
\hline LRM & $.998^{*}$ &, 432 &, 021 \\
\hline Capacitación $>$ 90 horas &, $332^{*}$ &, 155 &, 032 \\
\hline Constante & $-1,246^{*}$ &, 585 &, 033 \\
\hline R2 ajustado & & & .124 \\
\hline $\mathrm{n}$ & & & 1,499 \\
\hline${ }^{* * *} \mathrm{p}<.001 ;{ }^{* *} \mathrm{p}<.01,{ }^{*} \mathrm{p}<.05$ & & &
\end{tabular}

La falta de un mayor nivel de conocimientos tiene además un correlato de inequidad. Son las UGEL quienes tienen un mayor desafío territorial y una menor capacidad operativa quienes tienen, a nivel promedio, un menor conocimiento de sus funciones. Como se sabe, el MINEDU segmentó en 7 grupos a las UGEL según un doble índice, que representa la complejidad territorial que gestiona una UGEL y la capacidad técnica-operativa para realizar su tarea. En un extremo, las UGEL de tipo A son aquellas que tienen menor número de instituciones educativas rurales y unidocentes; distancias promedio más cortas entre UGEL y escuela; mayor presupuesto por IE; y menor ratio entre número de escuelas por especialista. En el otro extremo, las UGEL de tipo I, son aquellas que cuyas escuelas son en mayoría rurales y unidocentes, ubicadas a gran distancia de la UGEL, con bajo presupuesto y un número alto de escuelas por cada especialista (MINEDU, 2015c).

${ }^{13}$ A partir del cuadro se puede establecer el modelo de regresión logística para conocimiento de sus funciones:

$$
p_{(\text {conoce })}=1 / 1+e^{-\left(1.246+.573_{1} \text { Sexo }_{i 1}+.998_{p} L R M+.332_{p} \text { Capacitación }\right)}
$$


Cuando se analiza el conocimiento de funciones aplicado al personal según la tipología de UGEL, se encuentra un mejor conocimiento en aqueIlas UGEL que tienen un menor desafío territorial, pero a la vez una mejor capacidad operativa (43.8). Aquellas UGEL que tienen pobres condiciones tecnológicas, presupuestales y de infraestructura, con amplias extensiones de territorio, mayor porcentaje de escuelas rurales y dispersas, son UGEL cuyo personal tiene un menor nivel de conocimiento de sus funciones (gráfico 3).

Gráfico 3. Porcentaje promedio sobre Conocimiento de Funciones según Tipo de UGEL

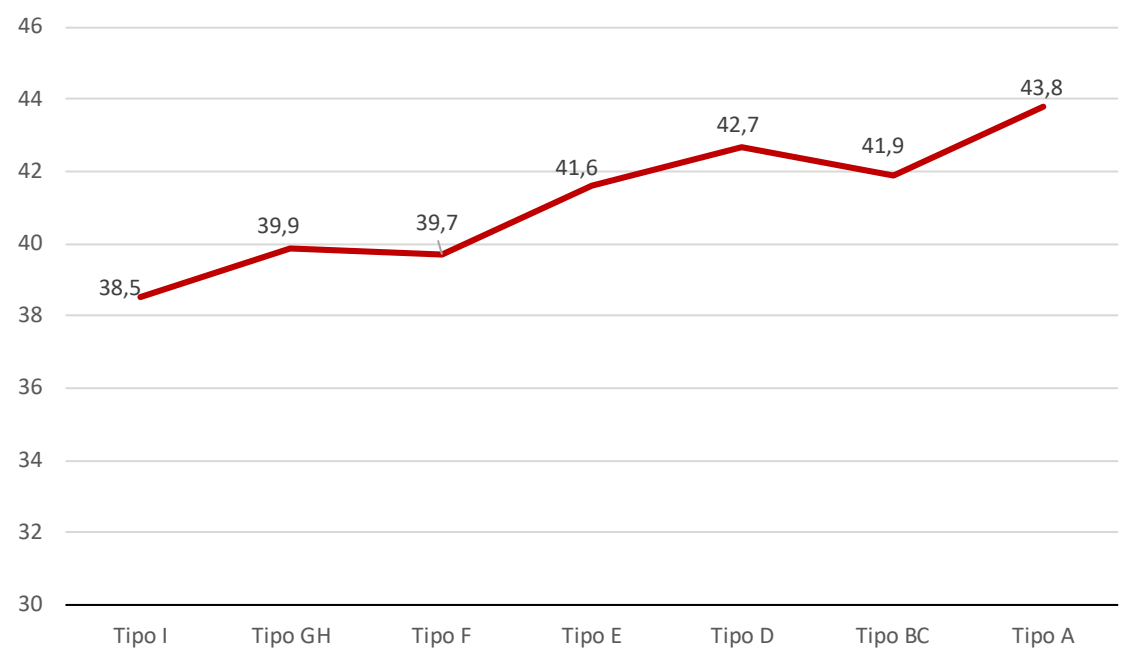

\section{Conclusiones y recomendaciones}

El actual proceso de descentralización en el Perú cumple 18 años. Del 2002 al 2005 se estableció un marco legal que permitió instaurar los gobiernos regionales, donde las DRE y UGEL pasaron a ser sus instancias de gestión educativa. La descentralización en educación ha tenido un enfoque de transferencias de competencias y funciones, principalmente entre los años 2007 y 2009 que fueron llamados como de "shock descentralizador". También se ha caracterizado por apuntar más a la participación que a la autonomía de sus instancias, creando consejos sectoriales de participación educativa que han sido afectados por su carácter voluntario y dependencia de la DRE o UGEL. En los últimos años, han existido esfuerzos por clarificar roles, simplificar trámites y establecer mecanismos de coordinación entre las UGEL, DRE y Ministerio de Educación, sin estar exentos de tensiones 
por miradas más centralistas de los gobiernos de turno (Ríos, 2016). En este contexto, el reto de sistemas unitarios como el peruano es encontrar sistemas de gobernabilidad y formas de gobernanza.

Desde una mirada de gobernabilidad, las UGEL tiene un problema de eficacia y calidad que se muestra en el débil conocimiento que tienen de sus funciones. Los resultados presentados demuestran que, a nivel nacional, el personal de las UGEL trabaja con un bajo conocimiento de sus funciones. El bajo conocimiento del personal de las UGEL se manifiesta en todas las áreas, pero con mayor fuerza en las áreas administrativas tales como racionalización, personal y presupuesto.

Aunque el $88 \%$ del personal manifiesta haber recibido capacitación, no ha tenido un mayor impacto en el conocimiento de sus funciones y posiblemente en el rendimiento o mejora de los servicios. Los importantes esfuerzos desplegados tanto por el ministerio de educación como los gobiernos regionales, que incluyen aportes propios de los servidores públicos como señalan Vegas (2016), Díaz, Valdivia y Lajo (2008) y The World Bank (2008) tienen problemas de eficacia y no se reflejan en los resultados esperados. En parte, como sostiene la PCM (2013) porque las capacitaciones se realizan de manera improvisada, atendiendo problemas inmediatos y sin ningún tipo de evaluación de resultados.

En ese sentido, elevar el rendimiento del personal de la UGEL, pasaría por refinar los mecanismos de capacitación y, en general, las políticas de desarrollo de capacidades. Los resultados del presente estudio convergen con varios autores en que la capacitación sí importa (Díaz, Valdivia y Lajo, 2008; Gonzales, Belaunde y Eguren, 2009). Sin embargo, depende mucho de la calidad y la duración. Por ejemplo, existen diferencias estadísticamente significativas a favor de aquellas capacitaciones que tienen como duración un número mayor de 90 horas. Esto hace suponer que no se requiere invertir tanto en talleres o jornadas de capacitación sino en especializaciones o diplomados, cuyos certificados deben estar vinculados a resultados en las funciones que tienen a cargo.

Además, la capacitación debería estar focalizando con personal de las áreas administrativas y de gestión institucional; en de ámbito rural y selva (tipo I, GH y F), con especial atención a regiones como Huánuco, Puno y Ucayali donde el nivel de conocimientos sobre las funciones a nivel regional refleja ser muy bajo.

Varios autores han observado que los docentes destacados en las UGEL han tenido problemas administrativos o disciplinarios, o no han podido desempeñarse bien en las escuelas (Valdivia y Díaz, 2008; The World Bank, 
2008). Por lo tanto, se podría suponer que el servicio que ofrecen o el nivel de conocimiento que tienen de sus funciones sea bajo o limitado. Sin embargo, los resultados muestran lo contrario. El personal docente destacado alcanza -relativamente- mejores resultados. Sin embargo, lo afirmado por los autores no necesariamente se contradice con los resultados encontrados en el presente estudio. Efectivamente, servidor que sigue un proceso administrativo en una escuela es ubicado temporalmente mientras dura el proceso en la UGEL, pero debe tenerse en cuenta que son casos puntuales. De ninguna forma se puede sustentar que un tercio del personal de la UGEL se encuentra en dicha situación. Buena parte del personal destacado son docentes que se encuentran en escalas remunerativas altas (47\% entre la cuarta y sexta escala), lo cual demuestra haber pasado por evaluaciones rigurosas de nivel nacional.

Ahora, si bien el conocimiento es un componente básico en el desarrollo de las competencias, no representa todos los elementos de una competencia para garantizar un comportamiento efectivo según la definición de Spencer y Spencer (1993). Como sostiene Alles (2005), los diagnósticos de conocimientos miden más memoria o dominio de la información cuando lo que importa es cómo se utiliza la información; los diagnósticos de conocimientos permiten evaluar si un funcionario discrimina una respuesta correcta, pero no si actúa de determinada manera; predicen lo que un servidor puede hacer, pero no lo que realmente hará. Y es aquí donde también importa poner atención a las motivaciones, rasgos y autoconceptos que tienen los servidores de UGEL.

Por otro lado, la gestión por competencias no se resuelve con diagnósticos de conocimientos y capacitaciones que reduzcan la brecha de conocimientos. La gestión por competencias demanda establecer un marco base de referencia que se utilice en los procesos de reclutamiento, selección, capacitación y desarrollo de personal, recompensas y otros aspectos de la gestión de los servidores públicos (IDS, 1997 en Op de Beeck y Hondeghem, 2010). En ese sentido, es un error buscar resolver la calidad del servicio que ofrece el personal de las UGEL sólo a través del desarrollo de capacidades. El ministerio debe funcionar más como un gestor de recursos humanos que uno de desarrollo de capacidades. Esto significa, brindar lineamientos de reorganización y perfiles de puesto, financiar nuevas plazas, asesorar sobre cuadros de personal, dimensionar el personal, inyectar meritocracia sobre todo en el área administrativa, fomentar la inclusión de gerentes públicos, revalorar el talento y la innovación, entre otras soluciones a corto y largo plazo; todo vinculado a las características del territorio o la tipología de UGEL vigente, y bajo el marco de la Ley Servir. 
Pero más importante, aun logrando resolver las brechas de conocimiento, y de la calidad y eficacia del servicio de las UGEL, no sería suficiente para satisfacer las demandas de los usuarios. Circunscribir las recomendaciones a un tema de eficacia, calidad, reducción de brechas de competencias y conocimientos sería lo que Aguilar (2010; 2007) Ilamaría un enfoque centrado en las capacidades del gobierno y no en las acciones de relación del gobierno con los usuarios, que son las que hacen reales, manifiestas, efectivas las competencias y conocimientos.

Desde un enfoque de gobernanza, se requiere el involucramiento de los sectores privados y sociedad civil local para crear lo que Del CastilloAlemán y Azuma (2011) Ilaman "redes políticas públicas. Siguiendo a Del Castillo-Alemán (2012), para que la UGEL alcance una gestión bajo la forma de gobernanza, se imponen cambios en cuatro direcciones: (i) Favorecer la participación y articulación de distintos actores (docentes, directores, padres de familia, municipio entre otros) en el proceso de diseño e implementación de proyectos; (ii) Generar nuevas formas de organización para la gestión que conduzcan a un sistema de relaciones menos jerárquico y más horizontal entre los actores; (iii) Revitalizar, en el proceso de toma de decisiones, el funcionamiento de órganos existentes como COPALE, mesas o comisiones especiales; (iv) Impulsar la construcción de redes entre los actores de la comunidad escolar y de la comunidad local, todo con el objetivo de generar condiciones para una acción colectiva en torno a la transformación de las escuelas.

Resistencia al cambio a este cambio puede ser natural y evidente; de hecho, no existe evidencia ni experiencia real y cercana de gobernanza en el sector educación que pueda estimular y orientar la experiencia. La situación se complejiza en contextos rurales donde resulta difícil que los grupos sociales carentes y dependientes de programas sociales del Estado se asuman como gestores de los programas y servicios; y puedan ir más allá de sus movilizaciones y quejas a fin de exigir respuesta a sus demandas (Aguilar, 2010). No obstante, sostiene el autor, son las localidades más pobres y vulnerables aquellas que no disponen de gobiernos eficaces; donde la UGEL muestra un limitado conocimiento de sus funciones como para que estén en condiciones de gestionar el servicio. 


\section{Bibliografía}

Aguilar, L. F. (2010). Gobernanza, el Nuevo Proceso de Gobernar. Ciudad de Mexico: Fundación Friedrich Naumann para la Libertad

Aguilar, L. F. (2007). El aporte de la Política Pública y de la Nueva Gestión Pública a la gobernanza. Revista del CLAD Reforma y Democracia, 39, 5-32.

Alles, M. A. (2005). Desempeño por Competencias, Evaluación de 360 . 1ra ed. 2da reimpresión. Buenos Aires: Gránica

Boyatzis, R.E. (1982). The Competent Manager: A Model for Effective Performance. Wiley: New York.

Boyatzis, R.E. (2008) Competencies in the 21st century. Journal of Management Developmen, 27(1), 5-12.

Curristine, T., Lonti, Z. y Joumard, I. (2007). Improving Public Sector Efficiency: Challenges and Opportunities. OECD Journal on Budgeting, 7(1).

Del Castillo-Alemán, G. (2012). Las políticas educativas en México desde una perspectiva de política pública: gobernabilidad y gobernanza. Magis, Revista Internacional de Investigación en Educación, 4(9), 637-652.

Del Castillo-Alemán, G. y Azuma, A. (2011). Gobernanza Local y Educación: La supervisión escolar. Ciudad de México: FLACSO México.

Defensoría del Pueblo (2013). Una mirada a la escuela rural. Supervisión a Instituciones Educativas Públicas de Nivel Primaria. Serie Informes de Adjuntía - Informe No 017-2013-DP/AAE. Lima: Defensoría del Pueblo

Defensoría del Pueblo (2017). El Derecho a la Educación. Supervisión nacional del proceso de matrícula e infraestructura escolar en las instituciones educativas públicas de nivel primaria. Informes de Adjuntía - Informe № 001-2017-DP/AAE. Lima: Defensoría del Pueblo

Díaz, H., Valdivia, N. y Lajo, R. (2008). Descentralización, Organismos Intermedios y Equidad Educativa: Un estudio de casos de las Unidades de Gestión Educativa Local - UGEL. En S. Gvirtz (Ed.) Equidad y niveles intermedios de gobierno en los sistemas educativos. Un estudio de casos en la Argentina, Chile, Colombia y Perú. Buenos Aires: Aique.

Dufour, G. (2008). El Rol (crítico) de los Actores de Nivel Intermedio en el Sistema Educativo Argentino: Un estudio de casos. En S. Gvirtz (Ed.) Equidad y niveles intermedios de gobierno en los sistemas educativos. Un estudio de casos en la Argentina, Chile, Colombia y Perú. Buenos Aires:Aique, 2008.

Emmerling, R. y Boyatzis, R. (2012). Emotional and social intelligence competencies: cross cultural implications. Cross Cultural Management,19(1), 4-18.

Ferrán, M. (1996). SPSS para Windows. Programación y Análisis Estadístico. Madrid: Mc Graw Hill.

García-Sáiz, M. (2011). Una visión constructiva de la gestión por competencias. Anales de Psicología, 27(2), 473-497.

Gil, J. (2007). La evaluación de competencias laborales. Educación XXI, 10, 83-106

Gonzales, N., De Belaunde, C. y Eguren, M. (2009). El Estado y la burocracia de los organismos intermedios del sector educación: un acercamiento desde los funcionarios. En: 
Montero, Carmen (ed.). El estado de la educación: estudios sobre política, programas y burocracia del sector, pp. 163-211. Lima: IEP.

Gvirtz, S. (2008). Equidad y niveles intermedios de gobierno en los sistemas educativos. Un estudio de casos en la Argentina, Chile, Colombia y Perú. Buenos Aires: Aique.

Hair, J., Anderson, R., Tatham, R. y Black, W. (1999). Análisis Multivariante. Madrid: Prentice Hall Iberia.

Hondeghem, A. y Op de Beeck, S. (2011). The government shift to competency management. Public Servants as Partners for Growth: Toward a Stronger, Leaner, and More Equitable Workforce. Chapt. 3. Paris: OECD Publishing.

Johnson, D. (1998). Métodos multivariados al análisis de datos. México DF: International Thomson Publishing

McClelland, D.C. (1973). Testing for Competencies rather than Intelligence, American Psychologist, 28, 1-14.

Ministerio de Educación del Perú - MINEDU (2017a). Plan de Trabajo del Equipo de Fortalecimiento de Capacidades 2017. Informe de la Dirección de Fortalecimiento de Capacidades, de la Dirección General de Gestión Descentralizada. Lima: Ministerio de Educación

Ministerio de Educación del Perú - MINEDU (2017b). Diagnóstico Situacional del personal en las DRE/GRE y UGEL, 2016 en PPT. Lima: Ministerio de Educación

Ministerio de Educación del Perú - MINEDU (2015a). Norma Técnica que regula la creación de las Unidades de Gestión Educativa Local en el ámbito Nacional, así como la inscripción en el Registro Nacional de Direcciones Regionales de Educación, o la que hagan sus veces, y de Unidades de Gestión Educativa Local - RENDUGEL. RVMGI 217-2015-MINEDU. 31 de Agosto, 2015. Lima: Ministerio de Educación.

Ministerio de Educación del Perú - MINEDU (2015b). Normas y orientaciones para el desarrollo del año escolar 2016 en la Educación Básica: responsabilidades de la DRE/GRE y las UGEL. R.M N 596-2015 MINEDU.

Ministerio de Educación del Perú - MINEDU (2015c). Lineamientos para la Gestión Descentralizada. RSG 938-2015-MINEDU. 31 de Diciembre, 2015. Lima: Ministerio de Educación.

Ministerio de Educación del Perú - MINEDU (2015d). Plan de Trabajo de las Acciones Formativas del Ministerio de Educación. Informe de la Unidad de capacitación en Gestión de la Oficina de Apoyo a la Administración Educativa. Lima: Ministerio de Educación.

Ministerio de Educación del Perú - MINEDU (2012). Reglamento de la Ley 28044 Ley General de Educación. DS 011-2012-EDU. Lima: Ministerio de Educación.

Nunes, F., Martins, L. y Duarte, H. (2007). Competency management in EU public administrations. Survey commissioned by the Portuguese EU-Presidency-EUPAN - Human Resources Working Group

Presidencia de Consejo de Ministros - PCM (2013). Política Nacional de Modernización de la Gestión Pública al 2021. DS N004-2013-PCM. Lima: PCM

Ríos, A. (2016). La ruta de la gestión descentralizada en la educación. Tarea, 96, 16-21. Disponible en https://bit.ly/3d5HMHr

OECD (2010). Strengthening government capacity en OECD. Reviews of human resource management in government: Brazil 2010: federal Government 
Op de Beeck, S. y Hondeghem, A. (2010). Managing Competencies in Government: State of the Art Practices and Issues at Stake for the Future.

SERVIR (2016). Diagnóstico de conocimientos al personal del Sistema Educativo Peruano. 010 y 011-2016-SERVIR/GDCRSC, Gerencia de Desarrollo de Capacidades y Rendimiento del Servicio Civil

SERVIR (2015). Los Diagnósticos de Conocimientos como Herramienta para el Desarrollo de Programas de Formación Basados en Evidencia. Documento de Trabajo. Sistemas Administrativos de Modernización de la Gestión Pública, Planeamiento Estratégico y Presupuesto Público. 7 de Julio de 2015

SERVIR (2010). Aprobación de Directiva que regula el desarrollo de los diagnósticos de conocimientos de las personas al servicio del Estado de los Sistemas Administrativos del Estado. Resolución de Presidencia Ejecutiva, 58 -2010-SERVIR/PE. Lima, 20 de Agosto, 2010

Shirazi, A. y Mortazavi, S. (2009). Effective management performance a competency-based perspective. International Review of Business Research Papers, 5(1), 1-10.

Siegel, S.y Castellan, N. J. (1995). Estadística no paramétrica: aplicada a las ciencias de la conducta (Vol. 4, pp. 195-196). México: Trillas.

SINEACE (2016). El desafío de construir calidad en las escuelas rurales del Perú: Experiencia en Julcán, La Libertad. Serie Estudios y Experiencias, 13. Lima: SINEACE

Spencer, L. M. y Spencer, S. (1993). Competence at work: Models for superior performance. Nueva York: John Wiley and Sons.

Sugimaru, C. y León, J. (2015). El éxito educativo de la región Moquegua, un análisis de la gestión pedagógica e institucional en tres niveles: regional, local y de institución educativo. Silex: Revista Interdisciplinaria, 5, 19-37.

The World Bank (2008). ¿Qué puede hacer un gobierno regional para mejorar la educación? Caso Junín. Lima: Tarea Asociación Gráfica Educativa, Lima- Perú

Valdivia, N. y Díaz, H. (2008). Diagnóstico de los organismos intermedios del sistema educativo peruano: un estudio de casos de las Unidades de Gestión Educativa Local UGEL. En: M. Benavides (ed.). Análisis de programas, procesos yresultados educativos en el Perú: contribuciones empíricas para el debate, pp. 275-295. Lima: GRADE.

Vegas, M. (2016). Entre la generación de alianzas y el trabajo con las personas: la experiencia de articulación institucional de la UGEL ISLAY (2011-2015). Publicación de Propuesta Ciudadana, agosto de 2016. 
Anexo 1. Porcentaje Promedio del Conocimientos de Funciones según Características del Personal

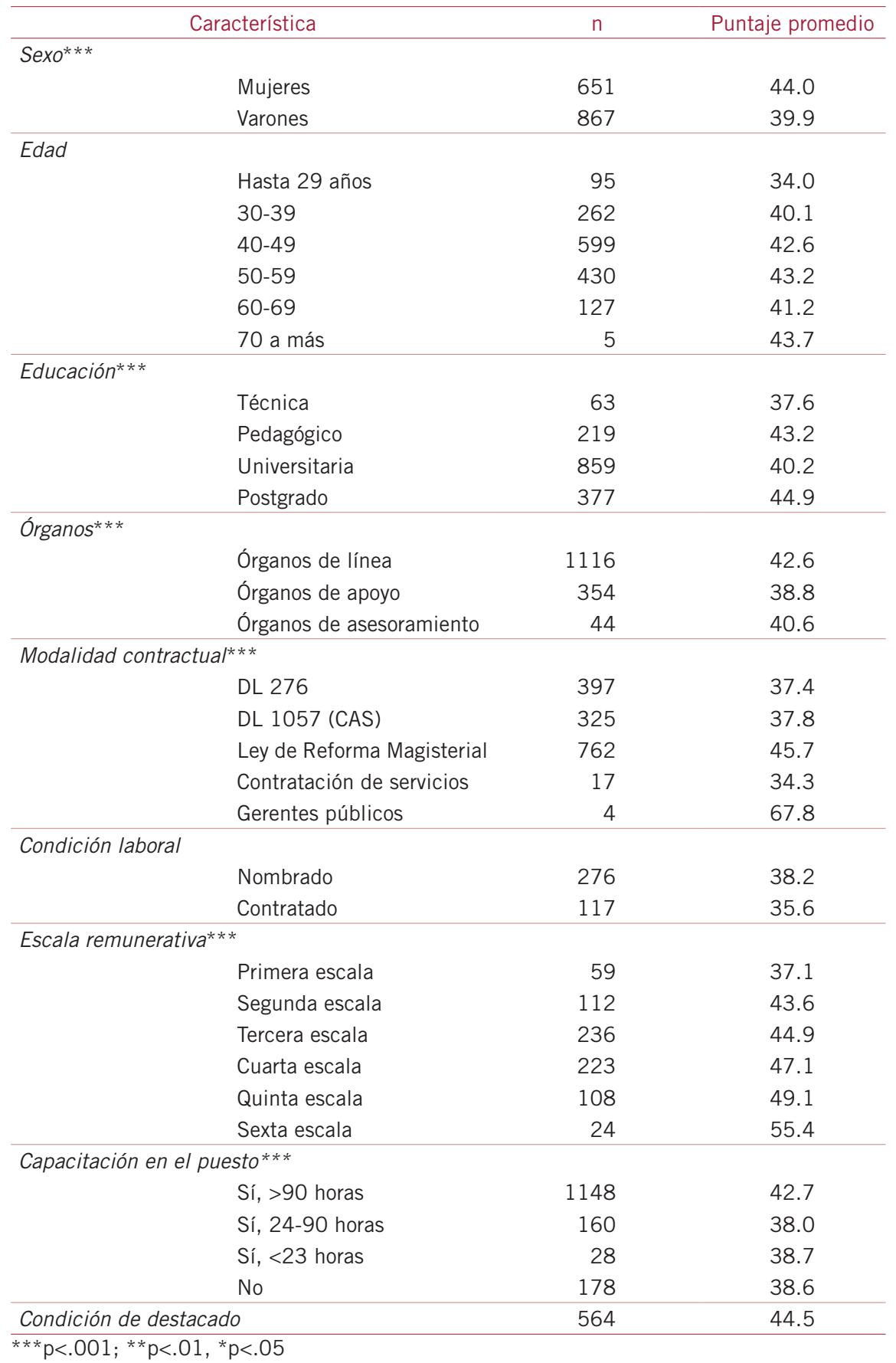


Anexo 2. Correlaciones de Características del Personal con Puntaje en Conocimiento

\begin{tabular}{|c|c|}
\hline Característica & r \\
\hline Ser varón & $-.140 *$ \\
\hline Edad & $.098 * *$ \\
\hline Educación & $.092 * *$ \\
\hline Experiencia general & $.164 * *$ \\
\hline Experiencia en servicio público & $.185^{* *}$ \\
\hline Experiencia en el puesto & $.052 *$ \\
\hline Ser docente & $.264^{* *}$ \\
\hline Ser administrativo & $-.186 * *$ \\
\hline Pertenecer a órgano de apoyo & $-.115^{* *}$ \\
\hline Pertenecer a órgano de línea & $.119 * *$ \\
\hline Ser CAS & $.143 * *$ \\
\hline Ser DL 276 & $-.195^{* *}$ \\
\hline Ser LRM & $.302^{* *}$ \\
\hline Ser nombrado & $.109 *$ \\
\hline Escala remunerativa docente & $.211 * *$ \\
\hline Capacitación & $.131^{* *}$ \\
\hline
\end{tabular}

${ }^{* * *} \mathrm{p}<.001 ;{ }^{* *} \mathrm{p}<.01,{ }^{*} \mathrm{p}<.05$ 